\title{
Crystal Structures and Viscoelastic Behavior in Different Morphologies of a Vinyl Alcohol-Ethylene Copolymer
}

\author{
María L. Cerrada, ${ }^{\dagger}$ Rosario Benavente, Ernesto PÉrez, and José M. Pereña \\ Instituto de Ciencia y Tecnología de Polímeros (CSIC), Juan de la Cierva 3, 28006 Madrid, Spain
}

(Received June 5, 2000; Accepted August 31, 2000)

\begin{abstract}
Different morphological structures of a vinyl alcohol-ethylene copolymer with high content (72 mol\%) in the former counit are obtained by various physical and chemical routes (annealing, stretching, and residual content of unhydrolyzed acetate groups). The specimens thus obtained are studied by X-Ray diffraction, differential scanning calorimetry and dynamic mechanical analysis in order to show the influence of the chemical, thermal, and mechanical histories on the crystalline structure, thermal properties and viscoelastic motions of the vinyl alcohol-ethylene copolymer.

KEY WORDS Vinyl Alcohol-Ethylene Copolymer / Crystal Structure / X-Ray Diffraction / Relaxation Mechanisms /
\end{abstract}

The growing use of vinyl alcohol-ethylene (VAE) copolymers with higher content in the former counit as barrier layers in the packaging of environmental sensitive products (mainly foods and pharmaceuticals) has led to a renewed interest in the studies of the parameters governing their crystalline structures and related properties, as the most adequate path to elucidate the final properties of those VAE copolymers. ${ }^{1,2}$ We have reported previously some papers on the composition, ${ }^{3,4}$ annealing, and orientation ${ }^{6,7}$ of several VAE copolymers, showing the role of each parameter on the structure and properties of the copolymers. In addition to those results, the aim of the present paper is to clarify the distinct morphologies obtainable in a single VAE copolymer, by either physical or chemical routes, and to show the consequences produced in the structure and properties by the morphological changes.

The various thermal histories imposed to different specimens of the VAE copolymer provoke changes in the contents of crystalline and amorphous phases, as well as in the crystalline lattice of the VAE copolymer. The latter effect can be also produced by drawing the original specimen due to a strain-induced phase change. In addition to this, the stretching process produces changes in the mobility of the macromolecular chains which are manifested in the presence of an additional viscoelastic relaxation. Moreover, the orthorhombic lattice of the quenched VAE copolymer can be distorted by the presence of small amounts of unhydrolyzed acetate groups.

All these results will be assessed and compared by different experimental techniques: wide-angle X-Ray diffraction, differential scanning calorimetry (DMTA) and dynamic mechanical thermal analysis (DSC), aiming to elucidate the changes in structure and properties produced by the different morphologies obtained from a VAE copolymer with high content (72 mol\%) in vinyl alcohol.

\section{EXPERIMENTAL}

\section{Specimens Preparation}

A commercially available random VAE copolymer (from Kuraray) has been used. Table I shows the composition in vinyl alcohol determined by means of ${ }^{1} \mathrm{H}$ and ${ }^{13} \mathrm{C}$ nuclear magnetic resonance (NMR) spectroscopies as well as in vinyl acetate groups determined by ${ }^{1} \mathrm{H}$ NMR spectroscopy. Sheets specimens were obtained as films by compression molding in a Collin press between hot plates $\left(210^{\circ} \mathrm{C}\right)$ at a pressure of $2.5 \mathrm{MPa}$ for $15 \mathrm{~min}$. Thicknesses of such films ranged from 200 to $250 \mu \mathrm{m}$.

The "parent" homopolymers, poly(vinyl alcohol), (PVAL), and polyethylene, (LDPE), were commercial grades and, for LDPE, the manufacturing conditions (pressure and temperature) were similar to those used for the production of the VAE copolymer studied. The PVAL homopolymer specimens were obtained by melt compression under the same conditions to those utilized for VAE copolymer. On the other hand, LDPE films were obtained by melt compression at $140^{\circ} \mathrm{C}$.

The different physical structures of the VAE copolymer have been achieved as follows:

1) Crystallization of the VAE copolymer was carried out under two different conditions: $Q$ and S (VAEQ and VAES specimens, respectively). The first treatment, $\mathrm{Q}$, consisted of a fast cooling between plates cooled with water after melting in the press. The $S$ specimen was slowly cooled from the melt, allowing the press to cool down after switching off the power. The corresponding cooling rates were, approximately, $100^{\circ} \mathrm{C} \mathrm{min}^{-1}$ for the $\mathrm{Q}$ sample and $2.5^{\circ} \mathrm{C}$

Table I. Vinyl alcohol (VA) and residual acetate groups compositions of the samples

\begin{tabular}{lccc}
\hline \multirow{2}{*}{ Sample } & \multicolumn{2}{c}{ Composition $\left(f_{\mathrm{VA}}\right)$} & \multirow{2}{*}{$f_{\mathrm{VAc}}$} \\
\cline { 2 - 3 } & ${ }^{1} \mathrm{H} \mathrm{NMR}$ & ${ }^{13} \mathrm{C} \mathrm{NMR}$ & ${ }^{1} \mathrm{H} \mathrm{NMR}$ \\
\hline VAE & 0.73 & 0.72 & $<0.0014$ \\
VAEAc & 0.73 & 0.72 & 0.027 \\
\hline
\end{tabular}

\footnotetext{
${ }^{\dagger}$ To whom correspondence should be addressed (Phone: 34-91-5622900, Fax: 34-91-5644853, E-Mail: ictcg26@fresno.csic.es).
} 


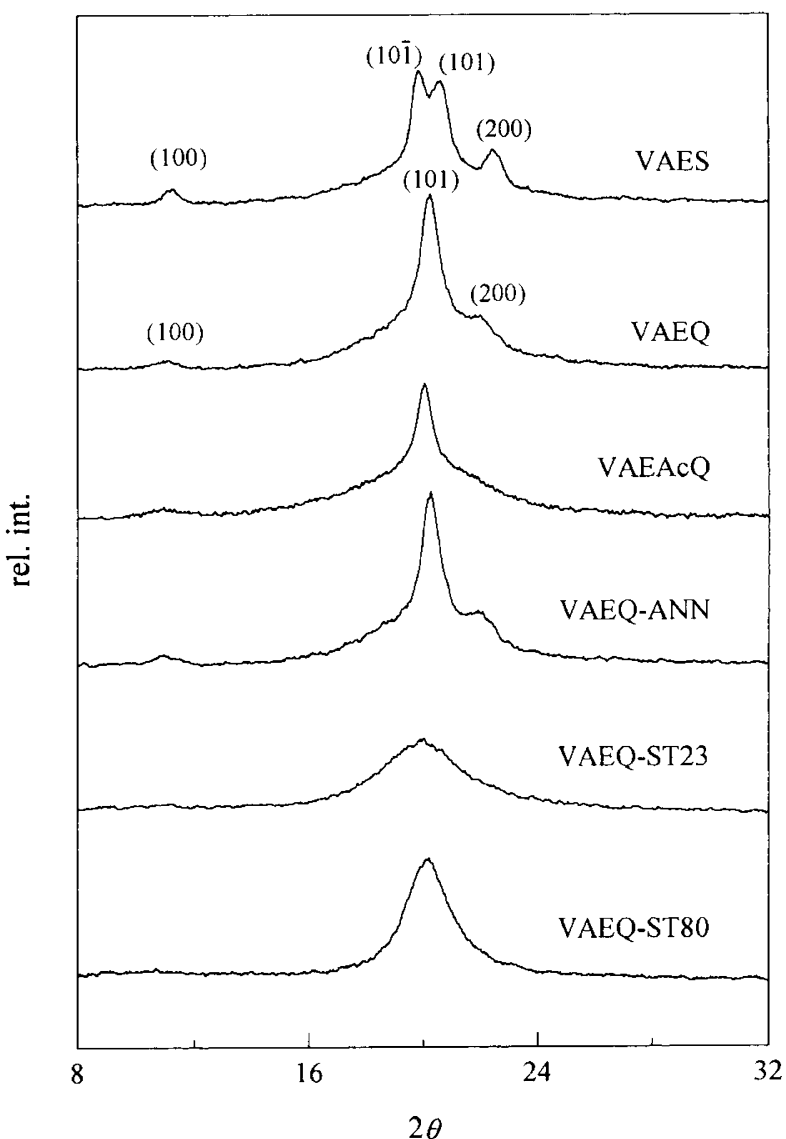

Figure 1. X-Ray diffraction patterns corresponding to the different specimens of the VAE copolymer. From the top to the bottom: VAES, VAEQ, VAEAcQ, VAEQ-ANN, VAEQ-ST23, and VAEQ-ST 80.

$\min ^{-1}$ for the $S$ one.

2) Thermal annealing at $80^{\circ} \mathrm{C}$ under vacuum imposed to VAEQ (VAEQ-ANN specimen). For that purpose, a specimen was placed in a desiccator for $8 \mathrm{~h}$ allowing it to cool down under vacuum overnight.

3) Saponification of the VAE copolymer performed under usual conditions ${ }^{1}$ into the corresponding vinyl acetate-ethylene copolymer and the subsequent hydrolysis, but not reaching the total conversion, i.e., some residual acetate groups, as shown in Table I, are present.

4) Uniaxial stretching process performed on VAEQ at two different temperatures: $23^{\circ}$ and $80^{\circ} \mathrm{C}$ (VAEQST23 and VAEQ-ST80 specimens, respectively). Dumb-bell shaped specimens with gauge dimensions $15 \mathrm{~mm}$ in length and $1.9 \mathrm{~mm}$ in width were punched out from the sheets with a standardized die. Tensile testing was carried out using an Instron Universal testing machine calibrated according to standard procedures. Specimens were drawn at a crosshead speed of $0.5 \mathrm{~cm} \mathrm{~min}{ }^{-1}$. The elongation of the samples is given by the draw ratio, $\lambda=l_{\mathrm{f}}$ $/ l_{\mathrm{o}}$, being $l_{\mathrm{f}}=$ final length and $l_{\mathrm{o}}=$ initial length. The actual draw ratio, $\lambda$, was directly measured on the samples from the spacing of parallel ink marks initially $2 \mathrm{~mm}$ apart. Draw ratio in the sample stretched at $23^{\circ} \mathrm{C}$ was about 3 , since this drawing temperature is below the glass transition and, consequently, failure occurs at relatively low draw ra- tios. On the contrary, the draw ratio attained was higher, around 6 , at the high stretching temperature $\left(80^{\circ} \mathrm{C}\right)$.

\section{Techniques}

Wide-angle X-Ray diffraction patterns were recorded in the reflection mode at room temperature by using a Philips diffractometer with a Geiger counter, connected to a computer. Ni-filtered $\mathrm{Cu}-K_{\alpha}$ radiation was used. The diffraction scans were collected over a period of 20 min between $2 \theta$ values from 3 to 43 degrees, using a sampling rate of $1 \mathrm{~Hz}$. The goniometer was calibrated with a standard of silicon.

The crystalline diffractions and the amorphous component have been separated with a fitting program which allows to estimate the crystallinity of the samples. The baseline has been taken just as a straight line in the $2 \theta$ range from 8 to 36 degrees, and no further correction has been applied. The different diffraction peaks were fitted to Voigt functions. The amorphous peak of the different samples was found to be centered at about $2 \theta=20$ degrees, based on our previous work. ${ }^{8}$ The error in the crystallinity determinations, when these are expressed as percentage, is estimated to be \pm 5 units.

Calorimetric analyses were carried out in a PerkinElmer DSC7 calorimeter, connected to a cooling system and calibrated with different standards. The sample weights ranged from 5 to $7.5 \mathrm{mg}$, and the heating rate was $10^{\circ} \mathrm{C} \mathrm{min}-1$. The value of the glass transition was obtained from the midpoint of the heat capacity change.

Viscoelastic properties were measured with a Polymer Laboratories MK II dynamic mechanical thermal analyzer working in a tensile mode. The complex modulus and the loss tangent $(\tan \delta)$ of each sample were determined at 3,10 , and $30 \mathrm{~Hz}$ over a temperature range from -150 to $150^{\circ} \mathrm{C}$, at a heating rate of $2^{\circ} \mathrm{C} \min ^{-1}$. The apparent activation energy values were estimated according to an Arrhenius-type equation, employing an accuracy of $1^{\circ} \mathrm{C}$ in the temperature assignment of $\tan \delta \max -$ ima.

\section{RESULTS AND DISCUSSION}

\section{Morphological Aspects and Thermal Properties of the Different Structures}

A detailed analysis of the crystalline structure in VAE copolymers has pointed out that both the composition and the thermal history are determining factors. ${ }^{8}$ Thus, previously reported maps of crystalline lattice $v s$. composition $^{9,10}$ displayed that a pseudohexagonal lattice is obtained for composition ranged between 20 and $60 \mathrm{~mol} \%$ vinyl alcohol (VAL) and that the crystalline regions are isomorphous to PVAL (monoclinic lattice) above $60 \mathrm{~mol} \%$ VAL. In the VAE copolymer under study ( $72 \mathrm{~mol} \%$ vinyl alcohol), it is found that VAES crystallizes into a monoclinic lattice as PVAL homopolymer, while VAEQ does show an orthorhombic-like crystal cell (Figure 1). Accordingly, the crystal lattice in VAES exhibits the same diffractions than PVAL homopolymer: (100), (10 $\overline{1}),(101)$, and (200) in the range of $2 \theta$ analyzed. However, due to the change in the crystal cell, the X-Ray pattern in VAEQ only shows the (100), (101), and (200) diffractions which are characteristic of the orthorhombic-like lat- 
Table II. Glass transition temperatures $\left(T_{\mathrm{g}}\right)$, melting temperatures $\left(T_{\mathrm{m}}\right)$, and enthalpies of melting $(\Delta H)$, lattice constants $(a, b, c$, and $\beta)$, Table II. Glass transition temperatures $\left(T_{\mathrm{g}}\right)$, melting temperatures $\left(T_{\mathrm{m}}\right)$, and enthalpies of melting $(\Delta H)$, lattice constants $(a, b, c, a$
densities of the crystal $\left(d_{\mathrm{c}}\right)$, and crystallinities deduced from the X-Ray diffractograms $\left(f_{\mathrm{C}}{ }^{\text {WXXD }}\right)$ of the different specimens

\begin{tabular}{|c|c|c|c|c|c|c|c|c|c|}
\hline Sample & $T_{\mathrm{g}}{ }^{\mathrm{DSC}} /{ }^{\circ} \mathrm{C}$ & $T_{\mathrm{m}} /^{\circ} \mathrm{C}$ & $\Delta H_{\mathrm{m}} / \mathrm{J} \mathrm{g}^{-1}$ & $a / \bar{\AA}$ & $b / \AA$ & $c / \AA$ & $\beta /$ degrees & $d_{c} / \mathrm{g} \mathrm{cm}^{-3}$ & $f_{\mathrm{c}}{ }^{\text {WAXD }}$ \\
\hline VAES & - & 183 & 75 & 7.90 & 2.53 & 5.29 & 92.28 & 1.221 & 0.47 \\
\hline VAEQ & 57 & 183 & 76 & 8.06 & 2.53 & 5.25 & 90.00 & 1.207 & 0.43 \\
\hline VAEAcQ & 53 & 169 & 51 & 8.12 & 2.53 & 5.29 & 90.00 & 1.191 & 0.27 \\
\hline VAEQ-ANN & 59 & 183 & 82 & 8.08 & 2.53 & 5.23 & 90.00 & 1.208 & 0.43 \\
\hline VAEQ-ST23 & - & - & - & - & - & - & - & - & - \\
\hline VAE-ST80 & 48 & 183 & 77 & 一 & - & - & - & - & - \\
\hline
\end{tabular}

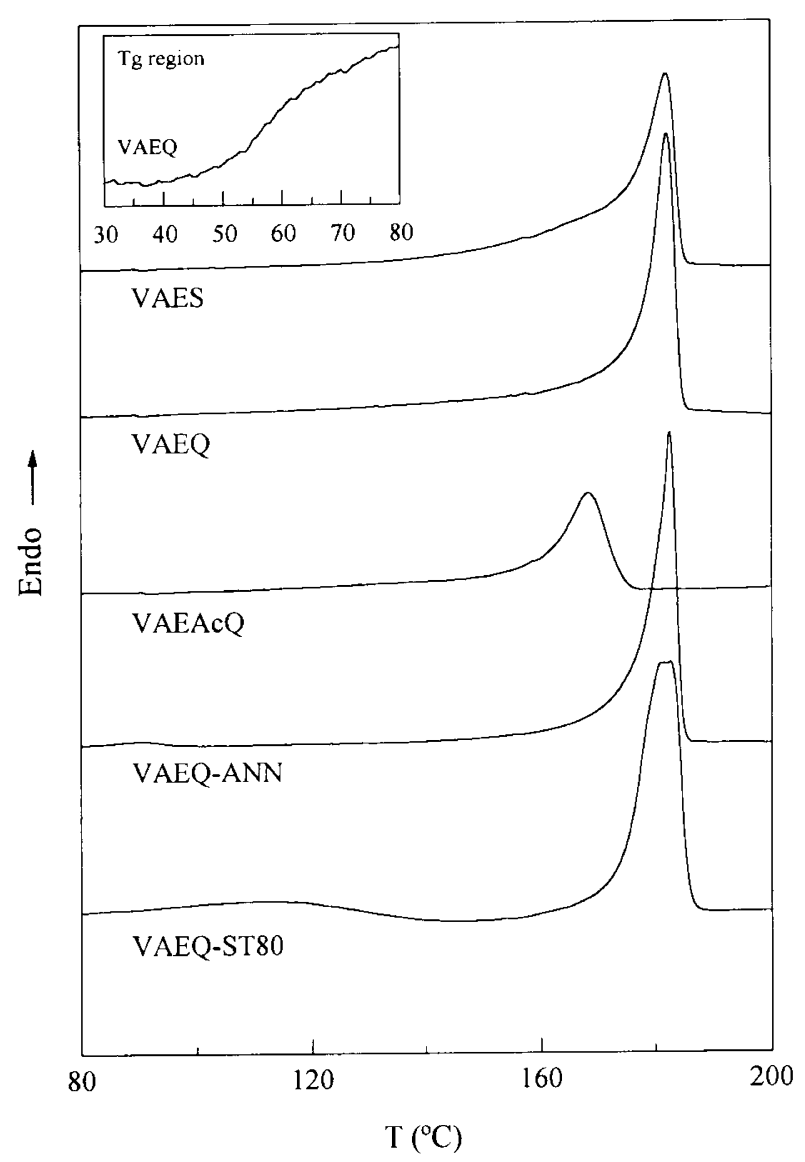

Figure 2. DSC curves corresponding to the different specimens of the VAE copolymer. From the top to the bottom: VAES, VAEQ, VAEAcQ, VAEQ-ANN, VAEQ-ST23, and VAEQ-ST80. In the insert, glass transition region for $V A E Q$.

tice ${ }^{8-10}$ It should be noted that the crystallographic conventions require that the chain axis is the $b$ axis in the crystal structure adopted by the distinct specimens of VAE copolymer under study.

Annealing does not affect the polymorphic behavior exhibited for VAE copolymers with either different composition or crystallization conditions. ${ }^{5}$ In the current case, VAEQ-ANN crystallizes into an orthorhombic-like lattice since it has been developed from the quenched VAE copolymer.

The morphological structure of this VAE copolymer is considerably modified due to the drawing process, as depicted in Figure 1. The WAXS patterns for the uniaxially drawn specimens (VAEQ-ST23 and VAEQ-ST80) have been recorded with the X-Ray beam parallel to the draw axis. The deformation process takes place through necking formation ${ }^{2,11}$ under the explored experimental condi- tions $\left(23^{\circ} \mathrm{C}\right.$ and $80^{\circ} \mathrm{C}$ as temperatures and $0.5 \mathrm{~cm} \mathrm{~min}^{-1}$ as crosshead speed) in the analyzed VAE copolymer. The distinct X-Ray diffraction patterns suggest a straininduced phase change. It can be observed in Figure 1 that the lattice presented by VAEQ is altered by drawing. On the one hand, a disappearance of the (200) diffraction and a broadening of the (101) one is observed at $23^{\circ} \mathrm{C}$ as stretching temperature. It seems that the $(200)$ reflection has merged into the main (101) displayed by VAEQ. Moreover, the (100) diffraction is broadened and becomes a diffuse peak, shifting to lower angles in relation to the unstretched VAEQ specimen. On the other hand, as the stretching temperature is raised at $80^{\circ} \mathrm{C}$ the main reflection becomes more intense than that observed in the stretched specimen at $23^{\circ} \mathrm{C}$, indicating that the orientation of the copolymer has increased as stretching temperature does. ${ }^{6}$ The strain-induced phase change, just pointed out, is consistent with the loss of lateral ordering judged from the vanishing of the (200) reflection and the broadening of the (100) one. An alike phenomenon has been found in vinyl alcohol-ethylene copolymers within the same composition range but under distinct empirical backgrounds. To explain this result, a transformation from the original undrawn lattice into a disordered crystalline form of mesomorphic character has been suggested. ${ }^{12,13}$ These common findings in this type of copolymers are in favor of a smectic-like order, as pointed out previously for uniaxially oriented polypropylene. ${ }^{14}$

VAEAcQ crystallizes into a crystalline cell similar to VAEQ (Figure 1), though diffractions are appreciably less sharper and poorly resolved as acetate residual groups have been introduced in the structure of the copolymer, suggesting a worse ordering (thinner crystallites). The (100) and, mainly, the (200) diffractions are the most affected by the non-hydrolyzed residual acetate groups. This feature points out that the $a$ crystallite axis is more modified by residual groups than the $c$ one, as viewed in Table II.

The separation of the diffractograms in the amorphous component and the crystalline diffractions allowed us to estimate the degree of crystallinity. The results presented in Table II show that crystallinity in VAES is higher than that exhibited by VAEQ. The slow crystallization process has allowed the formation of more and thicker crystallites. ${ }^{15,16}$ On the other hand, VAEQ-ANN exhibits a crystallinity analogous to that found inVAEQ, as listed in Table II. Changes in crystalline regions are not detected by this technique because results are within the experimental error range. Nevertheless, crystallites have been improved, as calorimetric measurements examined below point out (Figure 2 and Table II). In 


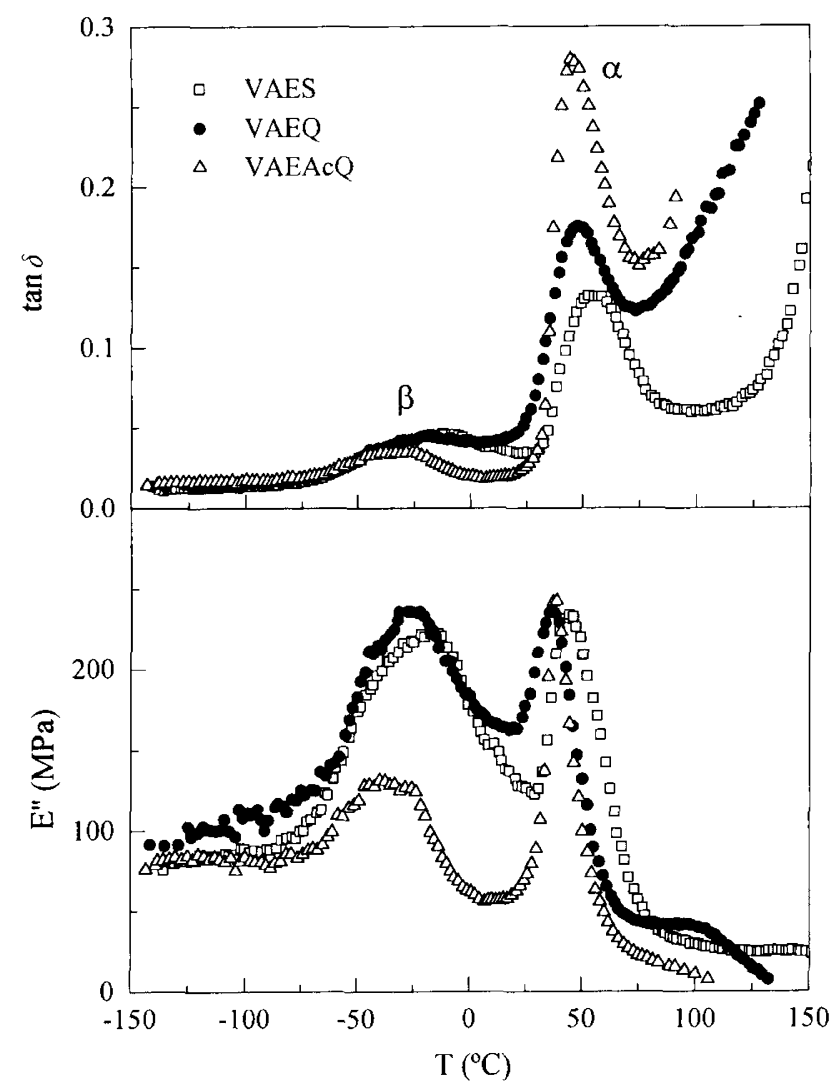

Figure 3. Temperature dependences of the loss tangent and the loss modulus corresponding to VAES, VAEQ, and VAEAcQ.

VAEAcQ, the results show that crystallinity decreases because of the restrictions imposed to crystallites formation by the presence of non-crystallizable acetate groups in the copolymer structure. The diminishment in crystallinity is in agreement with the decrease found in enthalpy for this specimen.

The DSC melting curves of the different specimens are displayed in Figure 2. The glass transition temperature of the different specimens are ranging within $50-60^{\circ} \mathrm{C}$ depending upon the developed structure, as depicted in the insert of Figure 2 for VAEQ and listed in Table II. A shift of the glass transition to higher temperatures is obtained in the annealed specimens since a slightly better development of crystallites has imposed on the annealed amorphous phase restraints greater than those present before the annealing. The glass transition temperature is also affected by the presence of the residual groups in VAEAcQ and, accordingly, it is moved to lower temperatures. PVAL homopolymer exhibits a glass transition temperature ${ }^{2}$ of $72^{\circ} \mathrm{C}$ by DSC. In such a polymer, the hydrogen bonds are very important, but the introduction of ethylene in vinyl alcohol-ethylene copolymers weakens those inter and intramolecular interactions and, consequently, provokes a shift of the glass transition toward lower temperatures. The presence of acetate groups causes an additional weakening of the originally strong hydrogen bonds and, therefore, the glass transition is shifted to even lower temperatures. In the current case, a $T_{\mathrm{g}}$ depression of around $4^{\circ} \mathrm{C}$ is observed. This feature points out the importance that those residual acetates have in the whole picture of properties though the amount of such groups is very small.
The melting temperatures, $T_{\mathrm{m}}$, of the crystalline phase are not clearly affected by crystallization conditions (VAEQ and VAES specimens) ${ }^{2,15}$ or by further annealing (VAEQ-ANN sample) or stretching (VAEQ-ST 80). However, a slight residual acetate content provokes a great $T_{\mathrm{m}}$ shift to lower temperatures, as seen in Figure 2 .

Moreover, Figure 2 shows a peak (or step) in VAEQANN displayed at a temperature about $13^{\circ} \mathrm{C}$ above the annealing temperature $\left(T_{\mathrm{a}}\right)$ though the main melting peak remains practically unaffected with annealing, as commented. The cause of this peak has been already pointed out in a comprehensive study performed at different $T_{\mathrm{a}}{ }^{\prime} \mathrm{s} .{ }^{15}$ The mentioned peak is due to the existence of material whose melting temperature is well below the main endotherm. Annealing seems to cause an improvement in the crystalline regions of VAEQ-ANN since the crystallites which melt at a determined temperature can crystallize in a later stage upon cooling from $T_{\mathrm{a}}$. Such crystallites are better developed than original VAEQ ones. In addition, an increase in melting enthalpy has been found in VAEQ-ANN due to those better formed crystals, as presented in Table II. It is also observed that the melting endotherm has been slightly narrowed as consequence of the annealing process. Therefore, the crystal size distribution has been slightly restricted.

In addition, Figure 2 shows the differences in the melting behavior between isotropic and stretched specimens. In VAEQ, a single peak is observed at the temperature range studied which corresponds to the melting of crystallites. On the contrary, a significant exotherm appears over the same temperature range for the specimen stretched at $80^{\circ} \mathrm{C}$ (VAEQ-ST80). This exothermic peak is ascribed to the reorganization of the mesomorphic phase into the orthorhombic one. It is worth noticing that an analogous exothermic transition has been observed for compression-drawn ${ }^{17,18}$ as well as quenched polypropylene. ${ }^{14,17,18}$ A first order smectic-to-crystal transition has been proposed ${ }^{14}$ to explain this feature.

A displacement to lower temperatures of the melting peak is observed in VAEAcQ, as mentioned above. In addition, a decrease in the melting enthalpy value is seen in Table II because of the residual acetate content. VAEQ has better formed crystals since it is totally hydrolyzed. The acetate groups are very voluminous and they become a hindrance to the crystallization process. Though the residual acetate content is very small, the melting enthalpy diminishment is considerable in VAEAcQ.

\section{Viscoelastic Behavior}

Three different relaxation processes $(\tan \delta$ results at 3 $\mathrm{Hz}$ ) have been described for the "parent" homopolymers. ${ }^{2,3}$ On the one hand, PVAL-Q shows relaxation mechanisms at $-53^{\circ}, 82^{\circ}$, and $113^{\circ} \mathrm{C}$, being named as $\beta, \alpha$, and $\alpha$ ' in order of increasing temperatures. On the other hand, the three viscoelastic relaxations found for LDPE-Q are located at approximately $-120^{\circ},-10^{\circ}$, and $37^{\circ} \mathrm{C}$, though the two latter processes are overlapped. In this case, the relaxations have been labelled $\gamma, \beta$, and $\alpha$, respectively.

On the contrary, only two different viscoelastic processes are exhibited in VAEQ, VAES, and VAEAcQ, at 
Table III. Relaxation temperatures, intensities ( $\tan \delta$ basis, at $3 \mathrm{~Hz}$ ) and activation energies for the different relaxation processes in the specimens

\begin{tabular}{|c|c|c|c|c|c|c|c|c|c|c|c|c|c|c|c|}
\hline \multirow{2}{*}{ Sample } & \multicolumn{5}{|c|}{$T /{ }^{\circ} \mathrm{C}$} & \multicolumn{5}{|c|}{$\tan \delta$} & \multicolumn{5}{|c|}{$\Delta H / \mathrm{kJ} \mathrm{mol}^{-1}$} \\
\hline & $\gamma$ & $\beta^{\prime}$ & $\beta$ & $\alpha$ & $\alpha^{\prime}$ & $\gamma$ & $\beta^{\prime}$ & $\beta$ & $\alpha$ & $\alpha^{\prime}$ & $\gamma$ & $\beta^{\prime}$ & $\beta$ & $\alpha$ & $\alpha$ \\
\hline PVAL-Q & - & - & -53 & 82 & 113 & - & - & 0.02 & 0.36 & 0.32 & - & & 50 & $>400$ & 300 \\
\hline VAES & - & - & -17 & 56 & - & - & - & 0.05 & 0.13 & - & - & - & 105 & $>400$ & 一 \\
\hline VAEQ & - & - & -21 & 48 & - & - & - & 0.05 & 0.18 & - & - & - & 110 & $>400$ & - \\
\hline VAEAcQ & - & - & -34 & 43 & - & - & - & 0.04 & 0.28 & - & - & - & 80 & $>400$ & - \\
\hline VAEQ-ANN & - & -50 & -6 & 80 & 121 & - & 0.03 & 0.04 & 0.12 & 0.15 & - & 60 & 110 & $>400$ & 250 \\
\hline VAEQ-ST23 & - & - & -15 & 48 & 107 & - & - & 0.07 & 0.18 & 0.17 & - & - & 95 & $>400$ & 280 \\
\hline VAEQ-ST80 & - & - & -14 & 41 & 132 & - & - & 0.07 & 0.09 & 0.10 & - & - & 95 & $>400$ & 210 \\
\hline LDPE-Q & -122 & - & -8 & 38 & 一 & 0.05 & - & 0.13 & 0.25 & - & 60 & - & 230 & 105 & - \\
\hline
\end{tabular}

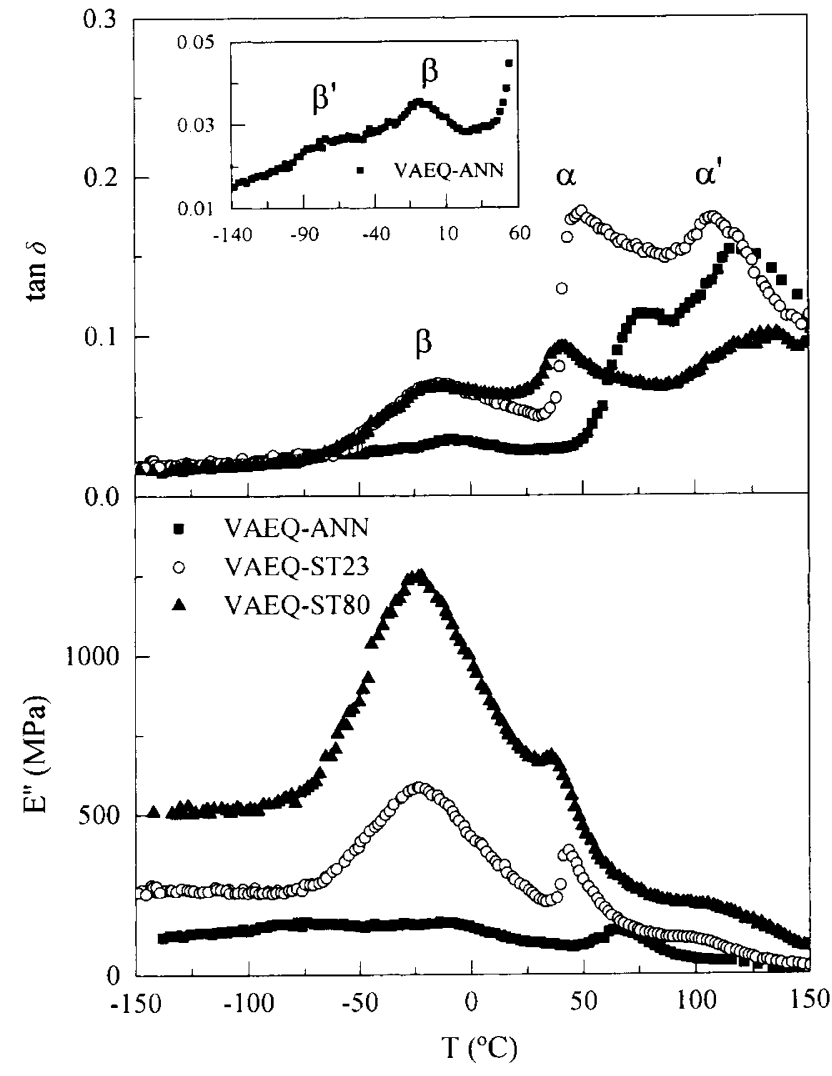

Figure 4. Temperature dependences of the loss tangent and the loss modulus corresponding to VAEQ-ANN, VAEQ-ST23, and VAEQ-ST80. In the insert, $\beta$ ' and $\beta$ relaxations region for VAEQANN.

approximately $-25^{\circ}$ and $50^{\circ} \mathrm{C}$ in loss tangent, as depicted in Figure 3 and listed in Table III. These peaks are called as $\beta$ and $\alpha$, as referred previously. ${ }^{2,19}$

Annealing has introduced some modifications in the structure of either amorphous or crystalline regions, as commented above. Such changes have a remarkable effect on the viscoelastic behavior of VAEQ-ANN. Thus, two additional relaxation mechanisms are exhibited in this annealed specimen under study, as shown in Figure 4. They have been labelled as $\alpha^{\prime}$ and $\beta$, respectively. The $\alpha$ ' process appears at a temperature higher than the $\alpha$ process. On the other hand, the $\beta$ ' mechanism is located at a temperature lower than the $\beta$ relaxation.

The structural changes provoked by the deformation process significantly affect the viscoelastic behavior exhibited in the stretched specimens. Thus, an additional

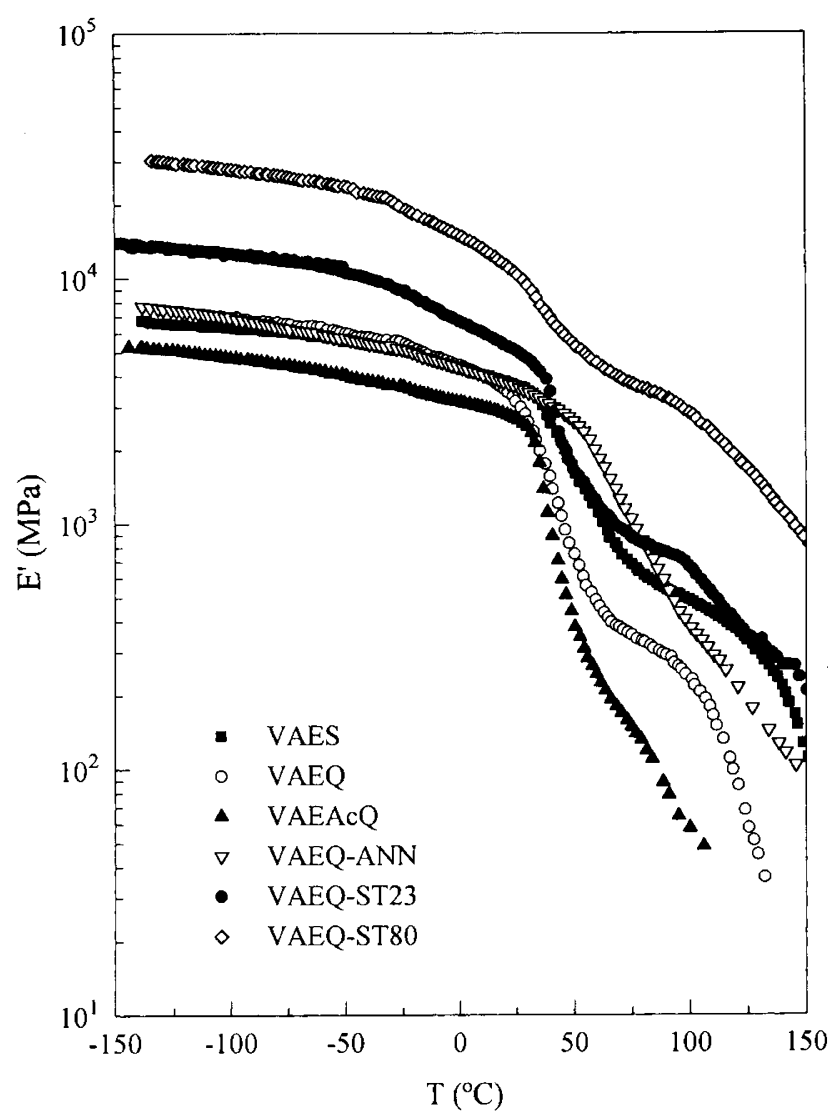

Figure 5. Temperature dependence of the storage modulus of the different VAE copolymer specimens.

relaxation mechanism is observed in VAEQ-ST23 and VAEQ-ST80 specimens, as seen in Figure 4. It appears at higher temperatures than the $\alpha$ process and has been labelled as $\alpha$ ' relaxation, like in VAEQ-ANN.

Figure 2 has shown a slightly endothermic behavior in VAEQ-ANN at a temperature range well below the main melting enthoterm in VAEQ, VAES, and VAEAcQ. Such a behavior points out the melting of the crystallites with the smallest sizes. As a first approximation, the modulus, and consequently the mechanical resistance, above $T_{\mathrm{g}}$ is related to the degree of crystallinity and the crystal morphology because of the cross-linking and filler effect of the crystallites. The melting of the small crystals causes the decrease of storage modulus after the glass transition (see Figure 5). Such a diminishment is significantly important as hydrolysis degree is slightly re- 
Table IV. Storage modulus values in the glassy state and at a temperature above the glass transition in the specimens (at $3 \mathrm{~Hz}$ )

\begin{tabular}{lcc}
\hline Sample & $E^{\prime}{ }_{-100{ }^{\circ} \mathrm{C}} / \mathrm{MPa}$ & $E_{100^{\circ} \mathrm{C}}^{\prime} \mathrm{MPa}$ \\
\hline PVAL-Q & 7400 & 200 \\
VAES & 6300 & 490 \\
VAEQ & 6950 & 230 \\
VAEAcQ & 4750 & 60 \\
VAEQ-ANN & 6950 & 375 \\
VAEQ-ST23 & 12600 & 675 \\
VAEQ-ST80 & 28000 & 2850 \\
LDPE-Q & 2600 & \\
\hline
\end{tabular}

duced. Accordingly, modulus values found for VAEAcQ are much lower (Table IV). On the other hand, drawing produces alignment of the macromolecules along the stretching direction, and, consequently, the storage modulus considerably increases in VAEQ-ST23 and VAEQ-ST80 as compared with VAEQ, VAES, VAEQANN, and VAEAcQ (Figure 5). Such an increase is more remarkable as stretching temperature is $80^{\circ} \mathrm{C}$ since the draw ratio in specimens stretched at the lower temperature is around 3 whereas at $80^{\circ} \mathrm{C}$ is about 6 , and the orientation is higher in the latter specimen. ${ }^{6}$

The different relaxation processes in $\tan \delta$ curves are well described as composed by contribution of different Gaussian curves, one for each observed relaxation. Such a deconvolution does not have a theoretical basis that can explain satisfactorily the shape of the dependence of $\tan \delta$ (or loss modulus) on temperature, though some factors that can influence it are known. A method of curve deconvolution ${ }^{20}$ has been proposed to analyze the dynamic mechanical loss curves in the region of the glass transition of several polymers, confirming the validity of this empirical approximation. In addition, it was shown that a Gaussian function provided the best fitting. The summation of several Gaussian curves provides a very good overall fitting over the whole experimental range measured in the different physical structures of this VAE copolymer, being an useful tool to determine the peak position. Some important data representing the dynamic mechanical behavior of the investigated materials, such as temperature location, intensity and apparent activation energy of the relaxations, are listed in Table III. The relaxation processes observed are analyzed separately as follows.

\section{$\alpha$ 'Relaxation}

VAE copolymers are semicrystalline irrespective of composition. ${ }^{10}$ Consequently, they could be expected to show a crystalline relaxation mechanism at temperatures higher than the relaxation associated to the glass transition, since crystallinity developed by the different structures of this VAE copolymer is high. However, VAEQ, VAES, and VAEAcQ specimens did not exhibit such high temperature $\alpha$ ' relaxation in the $\tan \delta$ plot, as seen in Figure 3 . The $\tan \delta$ values started to increase after the $\alpha$ mechanism but no other relaxation process takes place under those particular experimental conditions. The melting of the smallest crystallites, commented above, may be responsible that the $\alpha$ ' relaxation cannot be observed in those samples without a further thermal or mechanical history (annealing or stretching) after the initial processing. VAEQ, VAES, and VAEAcQ specimens might not have sufficient mechanical strength for measurements to be performed at the appropriate temperature. Consequently, this $\alpha^{\prime}$ relaxation is not shown in those isotropic specimens in $\tan \delta$ plots. On the other hand, in loss modulus plots (linear scale) the $\alpha$ ' relaxation could be overlapped within the high temperature side of the $\alpha$ process for VAES and VAEAcQ whereas it seems to be observed in VAEQ.

In PVAL, such $\alpha$ ' relaxation has been assigned to motions within the crystalline regions, which can be caused either by the relaxation of hydrogen bonds connecting the hydroxyl groups in this phase ${ }^{21,22}$ or to movements close to the crystal lamellae surface. ${ }^{23}$ Though its molecular origin is different, LDPE also shows a relaxation process related to motions in its crystalline regions. ${ }^{24}$ It appears at lower temperatures than in PVAL (around 40 ${ }^{\circ} \mathrm{C}$ ) and it is labelled as $\alpha$ (See Table III).

The VAEQ-ANN, VAEQ-ST23, and VAEQ-ST80 specimens display this additional mechanical relaxation at temperatures well above the process associated to the glass transition ( $\alpha$ relaxation). As described for PVAL, the $\alpha$ 'relaxation accounts for the activation of molecular mobilities within the crystal phase $\mathrm{s}^{21,22}$ or close to the crystal lamellae surface, ${ }^{23}$ since the main comonomer in this VAE copolymer is vinyl alcohol. Certain structural changes have been produced by effect of either annealing or stretching, and the relaxation associated with motions in crystalline regions is now observed either in $\tan \delta$ and loss modulus plots, as depicted in Figure 4.

In VAEQ-ST23, this mechanism is occurring at temperatures lower than in either VAEQ-ANN or VAEQ-ST 80. In addition, its intensity is higher. The loss of lateral ordering makes easy the motion in the crystalline regions. In VAEQ-ANN, such a mobility is more hindered and, consequently, the $\alpha^{\prime}$ relaxation is shifted to higher temperatures and its intensity is diminished. Finally, in VAEQ-ST80, in spite of the loss of ordering found, the $\alpha$ ' mechanism is moved to even higher temperatures and its intensity is the lowest one, because of the higher orientation developed within chains by the drawing at $80^{\circ} \mathrm{C}$.

\section{$\alpha$ Relaxation}

The $\alpha$ relaxation is considered as the glass transition in the different specimens of the VAE copolymer under study, and in the PVAL sample, due to its intensity and to the correspondingly strong decrease of the storage moduli at the relaxation maximum (as depicted in Figures 5 and 6 ). The value of the apparent activation energy is very high $\left(>400 \mathrm{~kJ} \mathrm{~mol}^{-1}\right)$ estimated from either loss moduli or $\tan \delta$ values (as listed in Table III). It has to be said that the frequency dependence with temperature in this relaxation mechanism has been considered to follow an Arrhenius behavior though it is due to cooperative motions. This approximation can be made without a significant error since the analyzed frequencies are low enough to be fitted to such a linear behavior just mentioned. However, if measurements are carried out at higher frequencies, the linear dependence is not longer exhibited, due to the non-Arrhenius character of the process studied in a broad range of frequencies. The "steepness index", $m$, has been also evaluated ${ }^{25}$ from a 


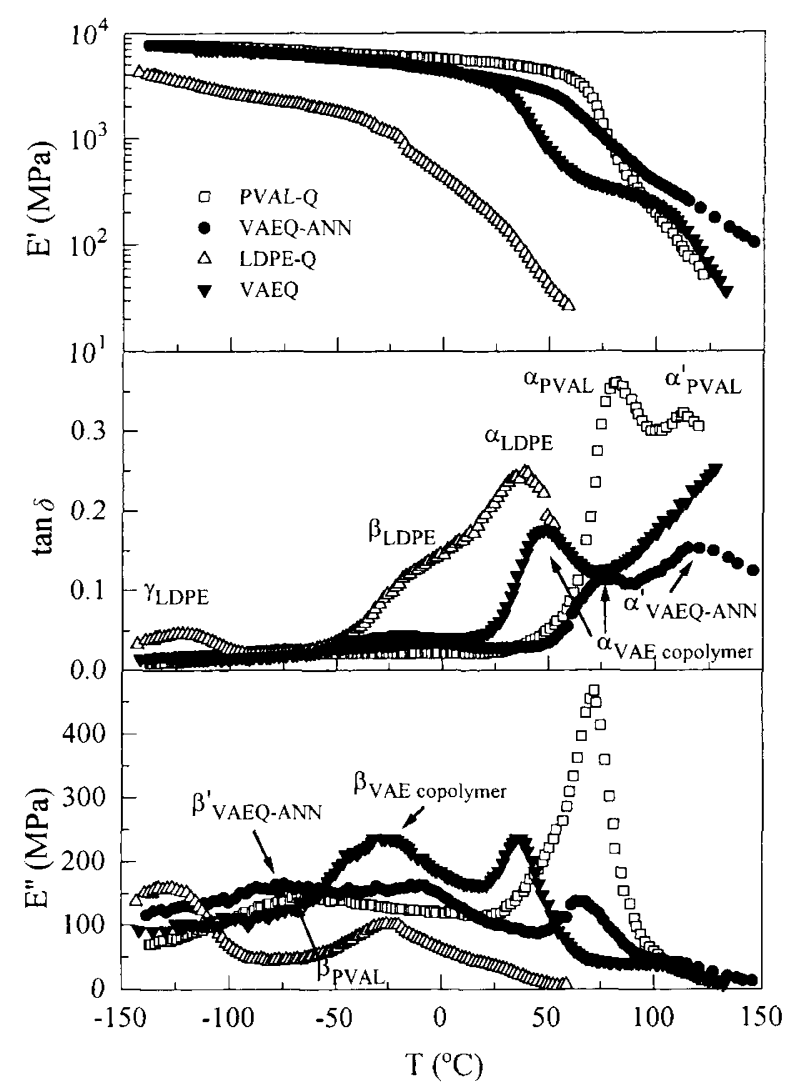

Figure 6. Temperature dependence of the storage and loss modulus and the loss tangent of quenched PVAL, VAEQ, VAEQANN, and quenched LDPE.

cooperativity plot (i.e., $\log \tau$ vs. $T_{\mathrm{g}} / T$ ). The $m$ values found in the different specimens of the VAE copolymer analyzed are much higher than 16 confirming the assignment of this $\alpha$ relaxation to the glass transition. In addition, the consideration of this relaxation process as associated to long-range motions taking place in the glass transition zone of the distinct VAE specimens agrees with the baseline change in the specific heat obtained in the calorimetric analysis. On the contrary, the mechanism associated to this relaxation in LDPE is different and it involves motions within crystalline regions, ${ }^{24}$ as commented above.

The temperature location of the $\alpha$ relaxation is dependent upon the structure of the VAE copolymer. The specimen slowly cooled from the melt, VAES, presents a higher crystallinity ${ }^{8,16}$ and larger and more perfect crystallites ${ }^{15,16}$ than the quenched, VAEQ, sample. Consequently, the glass transition temperature is shifted towards higher temperatures in VAES due to the major motion constraints imposed to the amorphous phase, where the relaxation process takes place. These mobility reductions also provoke a broadening in the relaxation times distribution and, consequently, the $\alpha$ relaxation process becomes broader, as observed in Figure 3. Moreover, the intensity of the $\alpha$ peak, considering $\tan \delta$ values, increases in VAEQ with respect to VAES, due to its major content in amorphous phase.

A small amount of residual acetate groups (VAEAcQ specimen) leads to a shift of the $\alpha$ process to lower temperatures (Figure 3). This feature is due to the major rigidity of vinyl alcohol in comparison to the acetate group as a consequence of the intra and intermolecular hydrogen bonds. The slight residual content of acetate groups makes difficult the establishment of hydrogen bonds and, consequently, the flexibility in the chains and the mobility are higher. Because this process is taking place in the amorphous regions and crystallinity is lowered with the residual content (Table II), the intensity of the $\alpha$ mechanism is higher in VAEAcQ than VAEQ, as seen in Figure 3.

Annealing induces changes within chains in either the amorphous or crystalline phases and the $\alpha$ relaxation process is affected. Therefore, the maximum temperature of this relaxation in the VAEQ-ANN specimen is moved to a much higher temperature and its intensity is considerably lower than that found in VAEQ.

The influence of stretching on the location and intensity of the $\alpha$ process is not apparent when drawing has occurred at $23^{\circ} \mathrm{C}$. Nevertheless, a shift to lower temperature is exhibited in VAEQ-ST80 as a consequence of the strain-induced mesomorphic change. Moreover, an important decrease is observed in the intensity of this mechanism due to a high alignment of chains in the stretching direction, as shown in the significant increase of modulus values found for this specimen (Figure 5 and Table IV).

\section{$\beta$ Relaxation}

The $\beta$ relaxation is very broad in all the samples analyzed. The different origins of this relaxation process in PVAL, LDPE, and the different VAE copolymer specimens should be noted. On the one hand, the $\beta$ mechanism in PVAL has been attributed either to hindered rotations of water molecules bound by hydrogen bonds to the polymer chains ${ }^{26,27}$ or to local torsion movements around main chain bonds. ${ }^{28}$ On the other hand, the $\beta$ relaxation has been universally detected in branched polyethylenes at temperatures around $-20^{\circ} \mathrm{C}$ but it sometimes appears, though weakly, in some samples of linear polyethylene. From the study of various polyethylenes and their copolymers, it has been concluded that this relaxation results from motions of chain units in the interfacial region. ${ }^{29,30}$ The temperature location of the $\beta$ peak and a higher value of the apparent activation energy suggest a mechanism for the VAE copolymers similar to that found in LDPE.

This relaxation mechanism is observed in VAES at a temperature slightly higher than in VAEQ because of the higher crystallinity in VAES. On the other hand, the $\beta$ process in the specimen with residual acetate groups (VAEAcQ) is shifted to lower temperatures and its intensity is diminished compared to VAEQ, as depicted in $\tan \delta$ and loss modulus plots in Figure 3. It seems, therefore, that the interfacial regions are reduced. Consequently, this relaxation is also narrower in VAEAcQ than in VAEQ, sample totally hydrolyzed.

The $\beta$ relaxation of VAEQ-ANN occurs at a temperature higher than that for specimens just discussed. This shift to higher temperatures is provoked by the order introduced in the chains during the annealing process. Moreover, the intensity of this $\beta$ relaxation has significantly diminished in VAEQ-ANN. In the stretched specimens, the $\beta$ process has been also moved to increasing temperatures because of the orientation introduced in 
the chains during the drawing process, though in a less extent than in VAEQ-ANN. In addition, the interfacial region content seems to have been increased due to the deformation process since the intensity of this $\beta$ relaxation has been raised.

\section{$\beta$ 'Relaxation}

This relaxation mechanism is only observed in VAEQANN. The annealing process, besides the shift of the $\beta$ relaxation to a higher temperature, leads also to the appearance of other new relaxation, which might be present in the original sample but overlapped to the $\beta$ mechanism. Figure 6 shows that this $\beta$ ' relaxation in VAEQ-ANN sample takes place in the same temperature range that the $\beta$ relaxation in PVAL. In addition, the apparent activation energy is rather similar in VAEQ-ANN and in the homopolymer, as displayed in Table III. Consequently, it seems to be caused by the same type of motions that the $\beta$ relaxation process in PVAL homopolymer. Thus, it could be attributed either to hindered rotations of water molecules bound by hydrogen bonds to the polymer chains or to local torsion movements around main chain bonds.

\section{CONCLUSIONS}

The type of the crystal lattice developed and the viscoelastic behavior are dependent upon the physical structure of the VAE copolymer. A monoclinic crystal cell is observed if the specimen is slowly crystallized from the melt while an orthorhombic-like structure is achieved in either the quenched or annealed samples. This lattice is distorted as a residual content of acetate groups remains in the structure. Finally, the stretched specimens exhibit a strain-induced phase change.

The main melting temperature is quite independent on the physical structure of this copolymer, excepting for the specimen with acetate groups not completely hydrolyzed, which displays a much lower melting temperature. On the contrary, the glass transition temperatures change with morphology.

Two relaxation processes have been observed in all the different specimens: $\alpha$ and $\beta$. Their temperature location and intensity are deeply affected by the structure. The $\alpha$ process is related to the glass transition of the different specimens whereas the $\beta$ one seems to be connected to the motion within the interfacial region. Moreover, the annealed (VAEQ-ANN) and stretched (VAEQST23 and VAEQ-ST80) specimens exhibit an additional $\alpha$ ' relaxation mechanism associated to the mobility within the crystalline phase. VAEQ-ANN also shows other relaxation process at temperatures lower than the $\beta$ one. The location and activation energy of this $\beta$ ' relaxation seems to indicate that its molecular origin could be either hindered rotations of water molecules bound by hydrogen bonds to the polymer chains or local torsion movements around main chain bonds, similar to the $\beta$ relaxation process in PVAL homopolymer.

Acknowledgment. The financial support of the CAM and the CICYT (Projects 07N/0051/1998 and MAT980961-C02-01) is gratefully acknowledged.

\section{REFERENCES}

1. A. S. Dunn, in "Polyvinyl Alcohol-Developments", C. A. Finch, Ed., John Wiley and Sons, London, 1992, p 195.

2. M. L. Cerrada, Ph. D. Thesis in Chemistry, Mechanical Properties, Viscoelastic Behavior and Anisotropy of Vinyl AlcoholEthylene Copolymers, Universidad Complutense, Madrid, 1995.

3. M. L. Cerrada, J. M. Pereña, R. Benavente, and E. Pérez, Polymer, 41, 6655 (2000).

4. M. L. Cerrada, R. Benavente, E. Pérez, and J. M. Pereña, J. Polym. Sci. Polym. Phys., 38, 573 (2000).

5. M. L. Cerrada, R. Benavente, E. Pérez, and J. M. Pereña, J. Polym. Sci., Polym. Phys., 29, 1 (2001)

6. M. L. Cerrada and J. M. Pereña, J. Appl. Polym. Sci., 64, 791 (1997).

7. M. L. Cerrada, R. Benavente, E. Pérez, and J. M. Pereña, Macromol. Chem. Phys., 201, 1858 (2000).

8. M. L. Cerrada, E. Pérez, J. M. Pereña, and R. Benavente, Macromolecules, 31, 2559 (1998).

9. T. Matsumoto, K. Nakamae, N. Ogoshi, M. Kawasoe, and H. Oka, Kobunshi Kagaku, 28, 610 (1973).

10. K. Nakamae, M. Kameyama, and T. Matsumoto, Polym. Eng. Sci., 19, 572 (1979).

11. M. L. Cerrada, R. Benavente, J. M. Pereña, and E. Pérez, Polym. Eng. Sci., 40, 1036 (2000).

12. K. Djezzar, L. Penel, J-M. Lefebvre, R. Séguelá, and Y. Germain, Polymer, 39, 3945 (1998).

13. L. Penel, K. Djezzar, J-M. Lefebvre, R. Séguelá, and H. Fontaine, Polymer, 39, 4279 (1998).

14. R. F. Saraf and R. S. Porter, Mol. Cryst. Liq. Cryst. Lett., 2, 85 (1985).

15. C. Fonseca, J. M. Pereña, R. Benavente, M. L. Cerrada, A. Bello, and E. Pérez, Polymer, 36, 1887 (1995).

16. E. Pérez, M. L. Cerrada, and D. L. VanderHart, J. Polym. Sci. Polym. Phys., 36, 2103 (1998).

17. R. F. Saraf and R. S. Porter, Polym. Eng. Sci., 28, 842 (1988).

18. S. Osawa and R. S. Porter, Polymer, 35, 545 (1994).

19. M. L. Cerrada, R. Benavente, J. M. Pereña, E. Pérez, and A. Bello, 'Dynamic Mechanical Behavior of Vinyl AlcoholEthylene Copolymers', in "Trends in Non-crystalline Solids", A. Conde, C. F. Conde, and M. Millan, Ed., World Scientific, Singapore, 1992, pp 321-324.

20. G. Rotter and H. Ishida, Macromolecules, 25, 2170 (1992).

21. M. Nagura, S. Matsuzawa, K. Yamaura, and H. Ishikawa, Polym. J., 14, 69 (1982).

22. P. D. Garrett and D. T. Grubb, J. Polym. Sci. Polym. Phys. Ed., 26, 2509 (1988).

23. P. D. Hong and K. Miyasaka, Polymer, 32, 3140 (1991).

24. I. M. Ward, "Mechanical Properties of Solids Polymers", 2nd ed, John Wiley and Sons, Chischester, UK (1985).

25. R. Boehmer, K. L. Ngai, C. A. Angell, and D. J. Plazek, J. chem. phys., 99, 4201 (1993).

26. K. H. Illers, Eur. Polym. J. -Supplement, 133 (1969).

27. W. J. Macknight and R. J. Tetreault, J. Polym. Sci. Part C, 35, 117 (1971).

28. T. Fujiki, M. Saito, M. Uemura, and Y. Kosaka, J. Polym. Sci., A-2, 8, 153 (1970).

29. R. Popli and L. Mandelkern, Polymer Bull., 9, 260 (1983).

30. R. Popli, M. Glotin, L. Mandelkern, and R. S. Benson, J. Polym. Sci, Polym. Phys. Ed., 22, 407 (1984). 